\title{
Progress in the physics of complex networks
}

\author{
G. Caldarelli ${ }^{1,2,3}$, G. Kaniadakis ${ }^{4}$, and A. Scarfone ${ }^{3,4}$ \\ 1 IMT, Institute for Advanced Studies, Piazza S. Ponziano, 6, 55100 Lucca, Italy \\ 2 London Institute for Mathematical Sciences, South Street 35a, Mayfair London, UK \\ 3 ISC-CNR, Dip. Fisica "Sapienza", Università di Roma, P.le A. Moro 2, 00185 Roma, Italy \\ 4 Politecnico di Torino, Department of Applied Science and Technology, Corso Duca degli \\ Abruzzi 24, 10129 Torino, Italy.
}

The present topical issue contains seven regular research papers, dealing with selected, hot themes of complex network physics, which have undergone the standard rigorous editorial process of EPJ-ST.

After years of research and many published papers, the theory of Complex Networks is still attracting increasing amongst colleagues and the general public [1-4]. The reasons for this success lie, in our opinion view, in the very general mathematical description that allows us to apply this theoretical framework to a variety of different situations [5-11]. Contrary to graph theory that has nevertheless provided most of the key quantities and concepts, here in complex network theory we make explicit use of self-similarity $[12,13]$. Quantities such as degree, betweenness, centrality, and assortativity are all described by fat-tailed frequency distributions if not clear power laws [14-18].

The presence of self-similar phenomena in Statistical Physics has a long tradition. From the classical studies of critical phenomena and renormalization group, we moved in the seventies and eighties of last century to the analysis of scale-invariance in the geometrical features of self-similar phenomena. A further evolution of this approach resulted in the analysis of scale-invariance in the time dynamics of these systems with concepts such as Self-Organized Criticality and studies of the so-called "sandpile models", an example of which is present in this issue. Most of the activity is now focussed on the search for scale-invariance in the topology, that is, in the way things are connected to each other.

In particular, in order to proceed with the study of the metabolic network that is one of the most important applications of the theory of networks, we need reliable stochastic models. The paper by C. Cianci et al. on autocatalytic reactions clearly shows that it is possible to characterize and quantify non-Gaussian fluctuations within the van Kampen descriptive scenario. In perspective, this work could, for instance, help in assessing the reliability of specific instances of metabolic networks.

The importance of simple stochastic models in the determination of the plausibility of a more general scenario is also at the basis of the paper by S. Caracciolo et al. Abelian Sandpiles are a typical example of simple stochastic models which generate long-scale space and time correlations. Again, the study of these classes of dynamics can help in describing time evolution in complex networks.

The dynamics of evolution in Complex Networks, for the specific case of metabolic networks (but also applied to economic growth), is also at the basis of a paper by A. De Martino et al. on Von Neumann's growth model. Thanks to the modification 
proposed in this paper, it is possible to theoretically understand the effect of various constraints on the cell machinery under the basic assumption of stability. These studies can clarify how and why we measure the metabolic networks instances.

Real Networks are indeed rather uncommon, and defy traditional symmetric approaches. In the paper by W. Miura et al. on business firm networks for instance, notwithstanding the common statistical properties, it is possible to observe a clear asymmetric behavior between the HUBS (large out-degree nodes) and the AUTHORITIES (large in-degree nodes). This fact, rather than being a topological issue, has deep consequences for the circulation of money in an economy. Again, simple stochastic models provide insight into what is going on in this phenomenon.

Information can be taken from networks by analyzing the spectral properties of the adjacency matrix. This is an important field of research with a long-standing tradition. In the paper by S. Maletic and M. Rajkovic, there is an important generalization to the concept of simplicial complexes that can encode more information in the links with respect to simple graphs. In perspective, this is particularly important for any social network system.

Coming back to the dynamics, one of the most studied of these structures is the analysis of epidemic spreading. By analyzing the SIS model with rewiring, in the paper by S. Wieland et al., the authors can assess the statistical properties of adaptive networks. In particular, the states of nodes and links remain substantially stable with respect to network evolution.

Finally we move to the paper by M. Cristelli et al. that throws some light on the structure of world trade. This network, intrinsically bipartite, reveals some information on the production rate and diversification of the different countries. Also, in this case, once the simple and linear relationships between the fitness of countries and the quality of products are given, spectral properties can be used to extract information.

In summary, complex network theory provides a completely new and interdisciplinary way to describe quantitatively a variety of systems only known in a qualitative fashion. This interdisciplinary feature is evident in this special issue where we have applications spanning from biology to economics describing catalytic reactions as well as the trade between nations or the business relationships amongst different financial institutions. At the same time, theoretical research in this field is proceeding towards a quantitative description of even more complicated systems such a network of networks, adaptive networks, or a more complex way to represent vertices interactions as in the theoretical contributions of the papers presented here. The importance of a network of networks became tragically evident during the Sigma Phi conference where these papers were presented. A major accident near the main electric plant of Cyprus took the lives of many persons and cut down electricity in the island. Disconnection of the electricity supply network disrupted (hopefully for a very short period, thanks to much effort) a variety of other services from transport to food distribution and electronic payments.

Many studies are still necessary to model and understand how to improve the robustness of infrastructural networks, but it is fair to say that in the smaller and more connected world that is expecting us in the near future, the science of networks will remain a necessary tool for taming the complexity of systems around.

The idea to publish some topical issues, one of which is the present, in peerreviewed international journals, collecting high-level, accurately selected, original research papers devoted to hot themes in statistical physics, was born during the 2011 Conference on Statistical Physics, held in Cyprus in July 2011. This idea immediately found a broad consensus within the conference sessions. For the considerable effort during the review process, we wish to warmly thank the referees along with all the other people who contributed in different ways to the preparation of this issue of EPJ-ST. 


\section{References}

1. S.N. Dorogovtsev, J.F.F. Mendes, Evolution of Networks: From Biological Nets to the Internet and $W W W$ (Oxford University Press, New York, NY, USA 2003)

2. T. Gross, H. Sayama, Adaptive Networks: Theory, Models and Applications (Springer, New York, 2009)

3. F. Harary, Graph Theory (Perseus, Cambridge, MA, 1995)

4. S. Boccaletti, V. Latora, Y. Moreno, M. Chavez, D.-U. Hwang, Phys. Rep. 424, 175 (2006)

5. R. Albert, A.-L. Barabási, Rev. Mod. Phys. 74, 47 (2002)

6. S. Bornholdt, K. Sneppen, Phys. Rev. Lett. 81, 236 (1998)

7. S. Brin, L. Page, Comput. Networks and ISDN Syst. 30, 107 (1998)

8. G. Kaniadakis, P.P. Delsanto, Math. Comp. Modell. 17, 31 (1993)

9. D. Dhar, Phys. Rev. Lett. 64, 1613 (1990)

10. P. Holme, G. Ghoshal, Phys. Rev. Lett. 96, 099701 (2006)

11. L. Pietronero, A. Vespignani, S. Zapperi, Phys. Rev. Lett. 72, 1690 (1994)

12. P. Bak, C. Tang, K. Wiesenfeld, Phys. Rev. Lett. 59, 381 (1987)

13. B.A. Huberman, L.A. Adamic, Nature 401, 131 (1999)

14. R.H. Atkin, Mathematical Structure in Human Affairs (Heinemann, London, 1974)

15. G. Kaniadakis, Eur. Phys. J. B 70, 3 (2009)

16. P.L. Luisi, The Emergence of Life (Cambridge University Press, Cambridge, 2006)

17. D. Gale, The Theory of Linear Economic Models (The University of Chicago Press, 1960)

18. N. Johnson, T. Lux, Nature 469, 302 (2011) 